\title{
DESARROLLO DE CONOCIMIENTOS Y HABILIDADES EN LA SOCIEDAD A TRAVÉS DE LA VINCULACIÓN UNIVERSITARIA
}

\author{
AUTORES: Kirenia Maldonado Zuñiga ${ }^{1}$ \\ Raquel Vera Velázquez ${ }^{2}$ \\ Edwin Joao Merchán Carreño ${ }^{3}$ \\ Franklin Jhimmy Tóala Arias ${ }^{4}$
}

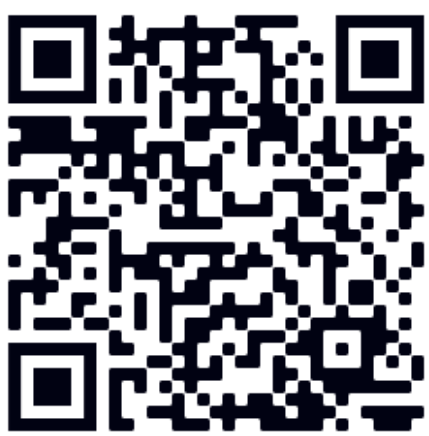

DIRECCIÓN PARA CORRESPONDENCIA:kirenia.maldonado@unesum.edu.ec

Fecha de recepción: 12/06/2019

Fecha de aceptación: 07/11/2019

\section{RESUMEN}

La presente investigación brindó un aporte significativo, dando a conocer la importancia de vincular los tres ejes esenciales: docencia, investigación y vinculación. En la Universidad Estatal del Sur de Manabí los estudiantes de la Carrera Tecnología de la información realizaron sus actividades de vinculación, con el objetivo de impartir capacitaciones de Ofimática Básica, dirigido a los Bomberos del Cuerpo de Guardia, estos desarrollaron conocimientos y habilidades informáticas que les permiten mejorar su vida laboral, por el giro tan avanzado que ha dado el uso de la tecnología en la mayoría de los trabajos es importante adquirir estos conocimientos. Las universidades generan estrategias y acciones con el fin de alcanzar un equilibrio entre el conocimiento la práctica y la investigación para formar estudiantes capaces de integrarse satisfactoriamente al proceso laboral. Los métodos utilizados fueron del nivel teórico, empírico y estadístico matemático. Los resultados evidenciaron la importancia de la vinculación universitaria. Se concluye que la vinculación universitaria con la sociedad es un factor necesario para capacitar al personal de la sociedad con el conocimiento de los universitarios. También se crearon las condiciones adecuadas para que los universitarios se ingresen al mundo laboral pasando el adiestramiento. De manera general ambos estudiantes se beneficiaron en la vinculación universidad con la sociedad. Se pudo confirmar que los estudiantes universitarios se motivan trasmitiendo sus conocimientos y pierden un poco el temor de introducirse al mundo laboral. Por otro lado se enriquece de conocimiento la sociedad fomentando un desarrollo interactivo entre universidad, docentes, estudiantes y sociedad.

\footnotetext{
1 Master en Ciencias de la Educación. Universidad de Ciencias Pedagógicas, Holguín, Cuba. kirenia.maldonado@unesum.edu.ec

2 Máster en Ciencias de la Educación. Universidad de Ciencias Pedagógicas, Las Tunas Cuba. raquel.vera@unesum.edu.ec

${ }^{3}$ Magíster en Docencia Universitaria e Investigación Educativa, Universidad de Loja - Ecuador. joao.merchan@unesum.edu.ec

${ }^{4}$ Magíster en Educación y Desarrollo Social. Universidad Estatal del Sur de Manabí-Ecuador. jhimmy.toala@unesum.edu.ec
} 
Kirenia Maldonado Zuñiga, Raquel Vera Velázquez, Edwin Joao Merchán Carreño...

PALABRAS CLAVE: adiestramiento; docencia; estrategias; impacto; sostenibilidad

\section{SOLID WASTE MANAGEMENT IN EDUCATIONAL INSTITUTIONS}

\section{ABSTRACT}

The present research provided a significant contribution, making known the importance of linking the three essential axes: teaching, research and linking. In the State University of the South of Manabí, the students of the Information Technology Career carried out their linking activities, with the aim of providing Basic Office training, directed to the Firemen of the Guard Corps, they developed knowledge and computer skills that They allow you to improve your working life, due to the very advanced turn that has taken the use of technology in most jobs it is important to acquire this knowledge. Universities generate strategies and actions in order to strike a balance between knowledge, practice and research to train students capable of successfully integrating into the labor process. The methods used were theoretical, empirical and mathematical statistical level. The results evidenced the importance of university bonding. It is concluded that the university relationship with society is a necessary factor to train the staff of society with the knowledge of university students. The appropriate conditions were also created for university students to enter the working world through training. In general, both students benefited from the university's association with society. It was confirmed that university students are motivated by transmitting their knowledge and lose a little fear of entering the workplace. On the other hand, society is enriched with knowledge, promoting interactive development between universities, teachers, students and society.

KEYWORDS: training; teaching; strategies; impact; sustainability

\section{INTRODUCCIÓN}

Actualmente las universidades desarrollan la vinculación con la sociedad, en el cual los estudiantes, docentes y sociedad cumplen un papel importante, cumpliendo con el objetivo de la formación teórica-práctica de los estudiantes y su inserción en las actividades laborales. Se establecen varias acciones y procesos académicos que se ponen al servicio de la sociedad, los estudiantes en formación pre profesionales obtienen sus conocimientos en las clases y los reproducen en las comunidades contribuyendo al mejoramiento de la calidad de vida de la sociedad. La universidad se planea proyectos de vinculación en función de un desarrollo más humanitario, solidario, equilibrado y respetuoso.

En las universidades a nivel mundial se le ha dado la importancia que tiene la vinculación universitaria con la sociedad. La actividad académica educativa no debería permanecer ajena a las necesidades de la sociedad, la vinculación universitaria con la sociedad es uno de los principios básicos en las Universidades. Contribuir con sus conocimientos al desarrollo de la sociedad es un objetivo.

Las universidades son el soporte para la solución de problemas de la sociedad a través de la vinculación universitaria, representando un beneficio que mejora la formación de los estudiantes y la calidad de vida de las sociedades, atendiendo tanto a la teoría como a la práctica. Uno de los temas desde la década del siglo XX.

Los estudiantes tienen como objetivo trabajar activamente en las problemáticas sociales aplicando los conocimientos que reciben en sus aulas para llevarlos a la práctica, motivándolos a involucrarse 
UNESUM-Ciencias: Revista Científica Multidisciplinaria UNIVERSITARIA

de manera activa y vivencial, palpando la realidad nacional y buscando estrategias de mejoramiento a la calidad de vida de los habitantes. El compromiso universitario se fundamenta en una estrategia motivacional hacia los estudiantes para que conozcan la realidad social de su entorno.

Las universidades deben fortalecer las funciones de servicio a la sociedad, erradicar la pobreza y el analfabetismo. Reforzar y ampliar las posibilidades de aprendizaje profesional, es importante combinar los estudios y trabajo, realizar intercambio de personal y planes de estudio mejor adaptados a la práctica profesional.

Las universidades necesitan crear acciones con el fin de alcanzar un equilibrio entre el conocimiento la práctica y la investigación que den como resultado estudiantes capaces de integrarse satisfactoriamente al proceso laboral, aplicando los conocimientos adquiridos par el desarrollo de las sociedades.

La universidad es la encargada de formar profesionales comprometidos con su localidad y con su país, en el cual pongan sus conocimientos y sus capacidades al servicio de la sociedad para solucionar sus problemas. La finalidad generar acciones satisfagan las necesidades de la sociedad, con el empleo justo y respetuoso de las personas. Las universidades como las organizaciones tienen el compromiso de atender necesidades sociales, por lo que sumar esfuerzos y lograr apoyar los objetivos para su cumplimiento es deber de todos.

El desarrollo de las sociedades depende del conocimiento de sus habitantes, los beneficios se muestran en la calidad de vida y en los ingresos económicos de la sociedad. En la última década lasa vinculación es una actividad que está presente en las universidades como objetivo principal, los conocimientos académicos en la solución de problemas, así como una participación en los sectores público y privado. Las universidades y su cumplimiento con la función de relacionarse con la sociedad y brindar profesionales experimentados a la comunidad que no estén ajenos a las necesidades sociales. Uno de los principios básicos de la universidad son los compromisos a cumplir con el deber, contribuir con sus medios al desarrollo de la práctica laboral, los estudiantes puedan analizar, proponer, experimentar y comprobar ideas y teorías que contribuyan a dar soluciones.

Las Instituciones de Educación Superior tienen la responsabilidad social de producir conocimientos, a través de la investigación, la educación superior tiene que ser de calidad para formar al recurso humano que necesita las sociedades, que responda a los diferentes retos del futuro, la producción de conocimiento y la formación del pre profesional. Dar soluciones de calidad a las necesidades sociales a través de la vinculación para aportar atención a las sociedades más vulnerables socio-económicamente.

El programa de vinculación en las universidades es una instrucción necesario para los estudiantes, de esta forma van interactuando con el campo laboral poniendo en práctica los contenidos adquiridos. Los pre profesional van tomando una postura con responsabilidad con respecto a la actividad laboral que los va preparando para cuando sean graduados, existen varios estudiantes graduados que se ven restringidos por la falta de experiencia y se les dificulta la incorporación en el mundo laboral, debido a esto se puede decir que la vinculación es una necesidad, por otra parte se cumple el objetivo fundamental de facilitar a la sociedad profesionales formados íntegramente para poner el conocimiento al servicio de la comunidad y la comunidad se beneficia al obtener el conocimiento trasmitido por los estudiantes. Hoy en día las instituciones desempeñan un rol importante estableciendo lazos que permitan el intercambio del conocimiento académico de los estudiantes y docentes con la sociedad. Es de gran importancia que cada institución educativa 
Kirenia Maldonado Zuñiga, Raquel Vera Velázquez, Edwin Joao Merchán Carreño...

establezca cuál es su compromiso con la comunidad en general, así se definen los avances que se proponen alcanzar a través de la vinculación. La relación universidad - sociedad.

La vinculación en la actualidad es una relación de interacción y beneficio recíproco entre las universidades y la sociedad, mejora la imagen de la universidad, la relación entre estudiantes, la eficacia del conocimiento que se adquiere, el servicio que se presta, la participación, capacitación y la creatividad de los estudiantes para un eficiente desempeño en el mundo profesional, permitiéndoles desarrollar capacidades, destrezas y actitudes que preparan al egresado para su desarrollo profesional. La relación entre el desarrollo profesional del estudiante y el mundo laboral es uno de los retos a seguir. En el desarrollo pre profesional la vinculación con la sociedad es el entrenamiento para ellos enfrentar el mundo laboral.

El aporte significativo que brinda la universidad a la sociedad desarrollando el conocimiento y provocando investigaciones innovadoras que contribuyen al mejoramiento de ambas partes, contribuir en el desarrollo local de manera permanente, a través de la vinculación universitaria es cumplir uno de los objetivos que se trazan las universidades de la actualidad.

Uno de los requisitos para la obtención del título es que los estudiantes deberán certificar servicios a la comunidad mediante prácticas o pasantías pre profesionales, debidamente monitoreadas, en los campos de su especialidad. Estas actividades se realizarán en coordinación con organizaciones comunitarias, empresas e instituciones públicas y privadas relacionadas con la respectiva especialidad. Además precisar para cumplir con la obligatoriedad de los servicios a la comunidad, brindándole los conocimientos necesarios para su desarrollo.

Uno de los objetivos de la vinculación universitaria es garantizar una formación de alta calidad que propenda a la excelencia y pertinencia del Sistema de Educación Superior, fortalecer la investigación, la formación académica y profesional, y la vinculación con la sociedad; articular la formación académica y profesional, la investigación científica, tecnológica y social, y la vinculación con la sociedad, en un marco de calidad, innovación y pertinencia; impulsar el conocimiento de carácter multi, inter y transdisciplinario en la formación de grado y posgrado, la investigación y la vinculación con la sociedad; desarrollar la educación superior bajo la perspectiva del bien para la sociedad, que les permitan el desarrollo del conocimiento y de la innovación.

Algunos servicios que se manifiestan en el sector productivo a las instituciones educativas: - Cursos de capacitación y adiestramiento. - Instalaciones para el desarrollo de proyectos con participación de estudiantes y docentes, el desarrollo de proyectos específicos. La vinculación ofrece el intercambio de conocimientos entre los estudiantes, docentes y la sociedad.

Los estudiantes de la carrera Tecnologías de la información de la Universidad Estatal del Sur de Manabí, apoyan los procesos de organización de las comunidades, con el fin de vincular las actividades académicas al estudio y solución de los problemas sociales y económicos, propician la innovación y uso del conocimiento científico y tecnológico para incrementar la productividad y competitividad, mejora el bienestar de la población en todas sus dimensiones, en concordancia con los planes de desarrollo institucional, y de la sociedad, la interacción académica mediante la cual la Universidad aporta a la sociedad, en forma crítica y creadora, los resultados y logros de la investigación y formación profesional.

La vinculación universitaria promueve la transferencia e intercambio del aprendizaje entre comunidad y universidad, vinculando los estudiantes y docentes a un contexto real a través de proyectos que generen impacto positivo especialmente en grupos vulnerables de la sociedad. La 
UNESUM-Ciencias: Revista Científica Multidisciplinaria

ISSN 2602-8166

CONOCIMIENTOS Y HABILIDADES EN LA SOCIEDAD A TRAVÉS DE LA VINCULACIÓN UNIVERSITARIA

universidad genera un cambio positivo en los procesos de desarrollo de las sociedades a través de la vinculación.

\section{DESARROLLO}

En el nuevo ordenamiento legal de Ecuador (Constitución, 2008) establece que el sistema de educación superior tiene como finalidad la formación académica y profesional con visión científica y humanista; la investigación científica y tecnológica; la innovación, promoción, desarrollo y difusión de los saberes y las culturas; la construcción de soluciones para los problemas del país, en relación con los objetivos del régimen de desarrollo, (art. 350). Sistema de educación que tiene que estar articulado al sistema nacional de educación y al Plan Nacional de Desarrollo (art 351).

La Universidad Estatal del Sur de Manabí concibe a la Vinculación con la Sociedad como el proceso sustantivo orientado a generar un sistema integrador entre la universidad y su entorno, para apoyar la investigación, la gestión académica y la docencia, a través de la ejecución de programas y proyectos multidisciplinarios de desarrollo, manifestados con enfoque sistémico, para problemas que presentan las sociedades, así se propicia el intercambio del conocimiento para generar un impacto positivo en el desarrollo sostenible de las sociedades y del país.

La vinculación con la sociedad le permite a los estudiantes realizar acciones de diversos géneros, lo cual es novedoso e interesante para ellos por ubicarse fuera de las actividades académicas formales de las instituciones, los estudiantes comparten sus conocimientos con la sociedad y se llevan la experiencia brindada por la comunidad. La vinculación con la sociedad constituye una función estratégica, permite enlazar la docencia con la investigación y difusión de sus saberes científicos, la formación integral de los estudiantes, los docentes y los participantes de la comunidad universitaria mantiene un ambiente de diálogo e intercambio de conocimientos, comprometidos con la sociedad.

La experiencia que viven los estudiantes en las aulas de la universidad son trasmitidas a la sociedad, este aporte significativo transforma la sociedad ofreciéndoles, interculturalidad, autodeterminación, pertinencia y desarrollo económico.

La Universidad Estatal del Sur de Manabí, como entidad académica superior, se ha convertido en una entidad con un rol que orienta a la identificación de necesidades y contribución a la solución de los problemas que existen en la sociedad, a través de la ejecución de proyectos de vinculación con la sociedad con pertinencia cultural, social y económica. La Vinculación con la Sociedad promueve y genera la transferencia de conocimientos particularmente a los sectores vulnerables.

La vinculación realizada por los estudiantes de la Carrera de Tecnologías de la Información y Agronomía, están inmersos en varios sectores como, salud, educación, cuerpo de bomberos, empresas entre otros, en las cuales han aportado conocimientos que les permiten a estas personas facilitar su trabajo así como mejorar los ingresos monetarios.

La comunicación activa y creadora con la sociedad, es decisiva para establecer prioridades y dar paso a una conciencia de cooperación mediante la cual los universitarios y los miembros de diversos sectores colaboren al desarrollo individual y colectivo de ambas.

Los estudiantes viven una experiencia que les permite desarrollarse enfrentando el mundo laboral, el cual les sirve como entrenamiento para el futuro. Además conocen los problemas de la su sociedad, lo cual los motiva a buscarle una solución. No existe mayor satisfacción que ver prosperar la sociedad y saber que el deber cumplido por parte de la vinculación universitaria, hace partícipe a todo el personal involucrado, Fortalecer las funciones de servicio de la sociedad, especialmente 
Kirenia Maldonado Zuñiga, Raquel Vera Velázquez, Edwin Joao Merchán Carreño...

aquellas orientadas a erradicar la pobreza, la intolerancia, la violencia y el analfabetismo es el deber de cada universidad.

Las instituciones de educación superior generar conocimientos y propuestas de solución a problemas estratégicos para el desarrollo y la sustentabilidad, socialización conocimientos para favorecer el intercambio de ideas y proyectos. Lo que permite las transformaciones en las sociedades. La universidad asume un papel relevante en este proceso. Aportando una educación para todos y para el futuro, favoreciendo a la educación, de jóvenes e individuos de todas las edades, los que podrán participar en los procesos de transformación social.

El impacto de la Universidad Pública en la sociedad está en fortalecer la capacidad de brindar a la sociedad soluciones a los problemas presentados, buscar la solución y ofrecerles una viabilidad que les permita su desarrollo. Los proyectos académicos se consolidan con el apoyo de un sólido programa de vinculación con la sociedad.

La vinculación con la sociedad es un factor de interacción estratégico de intervención en el territorio que hace posible responder y a la vez viabilizar la universidad con la pertinencia de su entorno, al precisar que educación superior debe responder a las expectativas y necesidades de la sociedad, la prospectiva de desarrollo científico, humanístico y tecnológico mundial.

Metodología

\section{Materiales y Métodos}

Los métodos utilizados en la investigación, manifestaron la importancia de la vinculación universitaria con la sociedad. El análisis demostró que las sociedades mejoran su calidad de vida y los estudiantes se preparan para enfrentar la vida laboral.

\section{MATERIALES Y MÉTODOS}

En la investigación se utilizaron métodos científicos tales como:

Del nivel teórico:

Histórico - lógico: se usó en la construcción de la investigación para la búsqueda de los antecedentes.

Análisis - síntesis: se utilizó para establecer criterios sobre vinculación con la sociedad.

Del nivel Empírico:

Entrevistas: brindó opiniones significativas para el desarrollo de la investigación.

Revisión bibliográfica: Se utilizó para la recopilación de información relacionada al tema, mediante libros, revistas de carácter científico, internet, entre otros

\section{Resultado}

Los resultados de la investigación demuestran la importancia de la vinculación universitaria con la sociedad. Los estudiantes adquieren sus conocimientos para luego trasmitirlos en los escenarios de vinculación, de esta forma solucionan diferentes problemas de la sociedad, lo que les permite la preparación para enfrentar el mundo laboral. Las sociedades desarrollan habilidades y conocimientos que les permiten el desarrollo en varios ámbitos, mejorando su calidad de vida.

\section{CONCLUSIONES}

64 UNESUM-Ciencias. Publicación cuatrimestral. Vol. 3, Año 2019, No. 3 (Septiembre - Diciembre) 
UNESUM-Ciencias: Revista Científica Multidisciplinaria

ISSN 2602-8166

CONOCIMIENTOS Y HABILIDADES EN LA SOCIEDAD A TRAVÉS DE LA VINCULACIÓN UNIVERSITARIA

Se concluye que la vinculación universitaria con la sociedad es un pilar necesario para contribuir al desarrollo de la sociedad, la interacción entre la universidad y la sociedad constituye una oportunidad para las instituciones de educación superior. Permitiendo la aproximación de las universidades y los colectivos sociales, para identificar las necesidades e inquietudes, estudiarlos y plantear soluciones a los problemas identificados, preservar los valores, la cultura y difundir los resultados de su trabajo. Los estudiantes ponen sus conocimientos al servicio de la comunidad, fomentando el desarrollo interactivo entre universidad, docentes, estudiantes y sociedad. La vinculación universitaria engrandece la educación superior.

\section{REFERÉNCIAS BIBLIOGRÁFICAS}

Abreu, O., Naranjo, M.E., Rhea B., Gallegos, M., Modelo Didáctico para la Facultad de Ciencias Administrativas y Económicas de la Universidad Técnica del Norte en Ecuador, doi: 10.4067/S0718-50062016000400002, Formación Universitaria, 9(4), 3-10 (2016) [ Links ]

Álvarez, F. F., El Modelo EFQM y su impacto en la calidad y la excelencia (en línea), 3 de septiembre de 2015, https://goo.gl/6JF9Bi, (2012)

Asamblea Nacional de la República del Ecuador, Ley Orgánica de Educación Superior, https://goo.gl/S2L9BF, (en línea), 20 de diciembre de 2016, Asamblea Nacional, Registro Oficial Año II, N² 298, 12 de octubre de 2010, Quito, Ecuador (2010)

Bautista, E. G., La importancia de la vinculación universidad-empresa-gobierno en México, https://goo.gl/oBMRN1, Iberoamericana para la investigación y desarrollo educativo ISSN 2007-7467,5(9), 1-2 (2014)

Campos, G. y Sánchez, G., La Vinculación Universitaria: Ese obscuro objeto del deseo, Electrónica de Investigación Educativa ISSN 1607-4041, 7(2), 11-12 (2005)

CEAACES. (2015). Adaptación del Modelo de Evaluación Constitucional de las universidades y Escuelas Politécnicas 2013 al Proceso de Evaluación, Acreditación y Recategorización de las Universidades y Escuelas Politécnicas 2015. Quito: CEAACES.

CEAACES. (2015). Modelo Genérico de Evaluación del Entorno de Aprendizaje de Carreras Presenciales y Semipresenciales de las Universidades y Escuelas Politécnicas del Ecuador (Versión Matricial). Quito: CEAACES.

Constitución de la República del Ecuador. 2008.

Dan, M-C., The Third Mission of Universities in the Development Strategy of Vienna City, https://goo.gl/ClKSbR, Informática Económica ISSN 1453-1305, 16(4), 49-56 (2012)

Etzkowitz, H., The entrepreneurial university wave: from ivory tower to Global Economic Engine, doi: 10.5367/ihe.2014.0211, Journal Industry and Higher Education, 28 (4), 223-232 (2014)

Fundación Europea para la Gestión de la Calidad (EFQM), ¿Qué es el modelo efqm? (en línea), https://goo.gl/T3UDDj, 3 de septiembre (2015)

Henningsen, B., Schlaeger, J. y Tenorth, H-E., Humboldts Model: The Future of the Universities in the World of Research: Conference Report, 1a edition, 201-231, BWV Verlag, Berlin, Germany (2013) 
Kirenia Maldonado Zuñiga, Raquel Vera Velázquez, Edwin Joao Merchán Carreño...

Gibbons, M., Limoges, C., Nowotny, H., Schwartzman, S., Scott, P., y Trow, M., The new production of knowledge. The dynamics of science and research in contemporary societies, 1st ed., Pomores-Corredos S.A., Barcelona, Spain (1997)

Gómez, M. y Escamilla, C., Modelo de vinculación entre las Instituciones de Educación Superior y las empresas: gestión del conocimiento, https://goo.gl/7niKgX, Rev. Acta Universitaria ISSN: 0188-6266, 22(2), 32-40 (2011)

González, M. y González, G., Extensión universitaria: Modelo teórico y de gestión para la educación superior cubana (en línea), 28 de agosto de 2015, https://goo.gl/0wMGZl, Ministerio de Educación Superior, Cuba (2006)

Larrea, E., Modelo de organización del conocimiento por dominios científicos, tecnológicos y humanísticos (en línea), 4 de mayo de 2015, https://goo.gl/9QA1Kg, Consejo de Educación Superior, Ecuador (2014)

Loi, M. y Di Guardo, M., The third mission of universities: An investigation of the espoused values, doi: 10.1093/scipol/scv012, J. Science and Public Policy, 42 (6), 855-870 (2015)

ITSO, I. T. (2015). Instituto Tecnlógico Superior Oriente. Recuperado el 25 de 03 de 2016, de PROYECTO INTEGRADOR DE GRADO: http://itsoriente.edu.ec/itso/index.php/proyectointegrador-de-grado

Martínez Rizo, F. (2000). Nueve retos para la educación superior. Funciones, actores y estructuras. México: Asociación Nacional de Universidades e Instituciones de Educación Superior.

Narro Robles, J. (2014). Retos de la universidad del siglo XXI. Revista Iberoamericana de Educación, 143145.

Palacios, M. G. (2009). Ejes transversales en el currículo de la formación inicial de docentes.CECC/SICA.

Peiró I Gregóri, S. (2013). Caracterización de la teoría pedagógica con relación a las ciencias de la educación: noción y lugar de la teoría pedagógica. RUA.UA.ES.ábal, M. V., \& Sanz, A. I. (1995). Los Ejes Transversales Aprendizajes para la Vida. Madrid: Escuela Española.

Rodríguez-Ponce, E. R. y Pedraja-Rejas, L. M., Percepciones sobre la Gestión del Conocimiento de Directivos Universitarios de Cuatro Universidades Chilenas, doi: 10.4067/S071850062016000400002, Formación Universitaria, 9(4), 41-52 (2016)

Vega-González, L. R., Modelo de gestión de proyectos de desarrollo tecnológico y vinculación de un centro de I\&DT universitario, Ingeniería Investigación y Tecnología ISSN 1405-7743, 12(1), 73-82 (2011)

Vizcaíno, J., Diseño de un Modelo de gestión para la vinculación de las instituciones de educación, Tesis de Magister, Facultad de Ciencias Económicas, Universidad Central del Ecuador, Quito, Ecuador (2013 